\title{
AJMALINE PROVOCATION TESTING FOR BRUGADA SYNDROME IN CHILDREN: THE GREAT ORMOND STREET EXPERIENCE
}

T G Day, M Bartsota, S Mead-Reagan, R Bryant, D Abrams, M Lowe, J Mangat, J P Kaski Great Ormond Street Hospital for Children

doi:10.1136/heartjnl-2013-304019.77

Background Brugada syndrome (BS) is a genetic disorder characterised by J point elevation in the right ventricular leads, ventricular arrhythmia and sudden cardiac death (SCD). The diagnostic ECG changes can be provoked by sodium channel blocking agents including ajmaline. Although ajmaline testing is well established in adults, there are few data on its safety and outcomes in paediatric cohorts3. Here we describe a single centre experience of ajmaline testing for BS in a large cohort of children.

Methods and results 100 consecutive patients aged 18 years or younger who underwent an ajmaline challenge between September 2004 and May 2012 were included. Mean age at the time of ajmaline challenge was 12.6 years (range $2.0-18.4$ years), and 54 were male. The indication for ajmaline challenge was a family history of 
BS in 49 patients, family history of unexplained sudden cardiac death (without a confirmed family history of BS) in 40, symptoms in 9 (syncope/collapse, $\mathrm{n}=5$; chest pain, $\mathrm{n}=1$; palpitations, $\mathrm{n}=3$ ), family history of long OT syndrome in 1 patient, and development of bradycardia with abnormal ECG during general anaesthesia in 1 patient. There were no serious adverse events associated with the ajmaline tests. A diagnostic type I response was seen in 19 individuals, of which 13 had a family history of BS, 5 had a family history of SCD without confirmed BS, and 1 patient had syncope as an indication for the challenge. Although a positive result was more common in those patients with a family history of BS, this association did not reach statistical significance. 5 individuals with a positive ajmaline test underwent electrophysiological studies; ventricular arrhythmias were not inducible in any. All patients are alive with no documented ventricular arrhythmias after a median follow-up time of 3.6 years.

Conclusions Ajmaline provocation testing in children is feasible and safe when performed in an appropriate setting by an experienced team. A type I response is more common in patients with a family history of BS, but the test may be helpful in identifying a diagnosis in families with unexplained SCD. Overall, BS appears to be associated with a very low risk of ventricular arrhythmia in the paediatric population. 